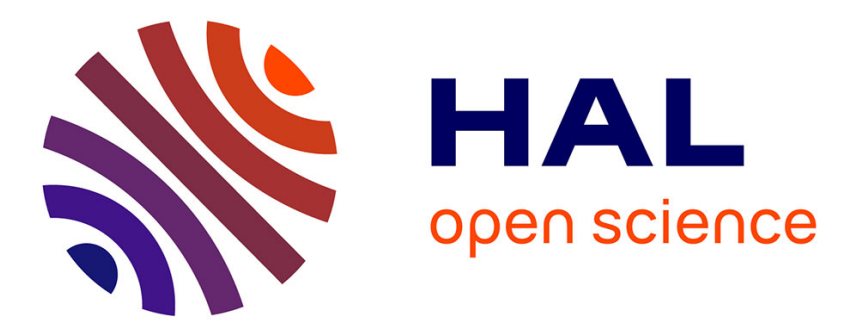

\title{
Quantum hydrodynamic model for the nonlinear electron dynamics in thin metal films
}

Nicolas Crouseilles, Paul-Antoine Hervieux, Giovanni Manfredi

\section{To cite this version:}

Nicolas Crouseilles, Paul-Antoine Hervieux, Giovanni Manfredi. Quantum hydrodynamic model for the nonlinear electron dynamics in thin metal films. Physical Review B: Condensed Matter and Materials Physics (1998-2015), 2008, 78, pp.155412. 10.1103/PhysRevB.78.155412 . hal-00596700

\section{HAL Id: hal-00596700 https://hal.science/hal-00596700}

Submitted on 30 May 2011

HAL is a multi-disciplinary open access archive for the deposit and dissemination of scientific research documents, whether they are published or not. The documents may come from teaching and research institutions in France or abroad, or from public or private research centers.
L'archive ouverte pluridisciplinaire HAL, est destinée au dépôt et à la diffusion de documents scientifiques de niveau recherche, publiés ou non, émanant des établissements d'enseignement et de recherche français ou étrangers, des laboratoires publics ou privés. 


\title{
Quantum hydrodynamic model for the nonlinear electron dynamics in thin metal films
}

\author{
N. Crouseilles \\ INRIA Nancy Grand-Est and Institut de Recherche en Mathématiques Avancées, 7 rue René Descartes, F-67084 Strasbourg, France \\ P.-A. Hervieux and G. Manfredi* \\ Institut de Physique et Chimie des Matériaux de Strasbourg, BP 43, F-67034 Strasbourg, France
}

(Received 25 June 2008; published 9 October 2008)

\begin{abstract}
A quantum hydrodynamic (fluid) model, derived from the Wigner-Poisson equations, is used to investigate the ultrafast electron dynamics in thin metal films. The hydrodynamic equations, which include exchange and correlation effects, can be combined into a single nonlinear Schrödinger-type equation. The fluid model is first benchmarked against a density-functional calculation for the ground state, with good agreement between the two approaches. The ultrafast nonlinear electron dynamics is then investigated and compared to recent semiclassical results obtained with a Vlasov-Poisson approach.
\end{abstract}

DOI: 10.1103/PhysRevB.78.155412

PACS number(s): 73.50.-h, 73.22.Lp, 05.30.Fk

\section{INTRODUCTION}

Understanding of the collective dynamics of a quantum electron gas is of great importance for a large variety of physical systems, including metallic nanostructures, thin metal films, semiconductor quantum wells and quantum dots. In particular, recent experimental progress in femtosecond pump-probe spectroscopy allowed the investigation of the electron dynamics on an ultrafast time scale, where collective effects play a crucial role. ${ }^{1-6}$ Thanks to the parallel development of advanced theoretical and numerical methods, ${ }^{7-10}$ meaningful comparisons between experimental and theoretical results are now becoming possible.

In typical pump-probe experiments, the electrons rapidly absorb the laser pulse energy and are brought out of equilibrium. This initial phase leads to the formation of collective oscillations (plasmon), which subsequently decay through coupling to the internal degrees of freedom of the electron gas (Landau damping). This damping occurs on a very fast time scale $(<10 \mathrm{fs})$ and was observed experimentally in gold nanoparticles. ${ }^{11,12}$ During these fast processes, the ionic background remains frozen and the electron distribution is nonthermal. As a result, the electron temperature cannot be properly defined at this stage of the relaxation process. On a longer time scale, the injected energy is redistributed among the electrons via electron-electron and electron-phonon collisions, leading to the eventual thermalization of the electron gas.

As the exact $N$-body dynamics cannot realistically be solved except for very small systems, ${ }^{13}$ it is necessary to develop appropriate reduced models, which, despite necessarily discarding some effects, still retain the principal physical features of the system under study. Most reduced models are improvements on the mean-field approximation (timedependent Hartree equations), whereby the motion of a single electron is determined by the overall distribution of charges (and possibly currents) in the system. The mean-field approach can be viewed as a zeroth-order approximation to the $N$-body problem in which two-body (and higher order) correlations have been neglected. Classically, this procedure is known as the BBGKY hierarchy, from the names of Bogoliubov, Born, Green, Kirkwood, and Yvon. ${ }^{14}$ Quantum models that go beyond the mean-field approximations comprise the Hartree-Fock equations (including exchange but neglecting correlations), density-functional theory (DFT, which retains both exchange and correlations in an approximate way), and various approaches based on the phase-space dynamics (Wigner and Vlasov equations).

All these approaches are rather costly from a computational point of view: the Hartree-Fock equations are nonlocal in space, DFT involves the resolution of $N \gg 1$ Schrödinger equations, and the Wigner-Vlasov approach requires the meshing of a six-dimensional phase space. Therefore, it would be useful to develop a reduced model that allows a more straightforward investigation of the collective electron dynamics.

For this purpose, a quantum hydrodynamic (QHD) model was recently developed ${ }^{15}$ by taking velocity moments of the Wigner phase-space distribution $f(x, v, t)$. Lower-order moments are related to physically relevant quantities such as the particle density, average velocity, and pressure. As for all hydrodynamic approaches, the moments obey an infinite hierarchy of equations, whereby the equation for the $i$ th order moment requires the knowledge of the $(i+1)$ th moment. Therefore, a closure assumption has to be made, which will depend on the particular conditions of temperature, density, and coupling to the environment. For metallic nanostructures the electron density is very high $\left(n \sim 10^{28} \mathrm{~m}^{-3}\right)$, yielding a Fermi temperature $T_{F} \sim 5 \times 10^{4} \mathrm{~K}$. Thus, for most practical purposes, metallic nanostructures can be thought of as operating effectively at zero electron temperature. This assumption allows us to establish a relationship between the electron pressure and density, thus closing the QHD system of equations. We note that these equations do include quantum effects through the so-called Bohm potential and thus fully deserve the qualification of "quantum hydrodynamics."

Hydrodynamic models were developed several times in condensed-matter physics, particularly for applications to semiconductors (Bloch equations) ${ }^{16}$ and, to a lesser extent, metal clusters, $7,17,18$ where they are frequently referred to as time-dependent Thomas-Fermi models. However, most hydrodynamic models used in the past do not actually contain quantum effects, apart from the Fermi-Dirac statistics. Further, the results of the hydrodynamic simulations were gen- 
erally validated for the electric-dipole response and frequency spectrum, ${ }^{17}$ whereas higher-order quantities (such as the thermal and potential energies) were not properly assessed. Recent numerical simulations ${ }^{19-21}$ and experiments ${ }^{22}$ have highlighted a number of interesting effects involving precisely these quantities when thin metal films are irradiated with femtosecond laser pulses-most notably, ultrafast heating and ballistic oscillations of the electron gas. It is the main scope of this paper to establish whether these subtler effects can be described with a hydrodynamic model.

There has also been a recent surge of interest in QHD models for gaseous quantum plasmas, ${ }^{23,24}$ in view of possible applications to the dense plasmas found in some astrophysical objects (white dwarfs, giant planets) and in experiments on inertial confinement fusion. ${ }^{25}$

The QHD model of Ref. 15 has the advantage of being rigorously derived from the Wigner-Poisson equations so that the underlying hypotheses, and hence the limitations of the model, are clearly spelled out. In particular, the two main limitations of the QHD model are that: (a) quantum effects are included only to second order in the Planck's constant and (b) wavelengths shorter than the Thomas-Fermi screening length $L_{F}$ are not treated properly. Here, $L_{F}=v_{F} / \omega_{\mathrm{pe}}$, where $v_{F}$ is the Fermi speed and $\omega_{\mathrm{pe}}$ is the plasmon frequency. The latter restriction is analogous to the well-known limitation for classical fluid models, which are valid for wavelengths longer than the Debye length (i.e., the typical screening length in a classical plasma).

In this work, we present a detailed comparison of the results of the QHD simulations against numerical results obtained with a semiclassical model based on the Vlasov equation. The classical results were well documented in a series of previous publications ${ }^{19-21}$ dealing with the ultrafast electron dynamics in thin metal films. We will show that, in spite of the above limitations, the QHD model is capable of reproducing some of the most notable results obtained with the Vlasov approach.

The rest of this paper is organized as follows. First, the QHD model is described in Sec. II. Then the ground state is computed and benchmarked against the ground state obtained with a DFT approach (Sec. III). In Sec. IV, the QHD model is used to simulate the nonlinear electron dynamics, and the results are compared to those obtained with the semiclassical model. Conclusions and possible further developments are reported in Sec. V.

\section{QUANTUM HYDRODYNAMIC MODEL}

The quantum QHD model used throughout this paper was derived in Ref. 15 starting from the Wigner-Poisson system and taking velocity moments of the Wigner pseudodistribution function. The Wigner representation ${ }^{26}$ is a useful tool to express quantum mechanics in a phase-space formalism (for reviews, see Refs. 27 and 28). In this representation, a quantum state-either pure or mixed-is described by a function of the phase-space variables $f(x, v, t)$, which obeys an integrodifferential evolution equation similar to the familiar Vlasov equation of classical plasma physics. The Wigner function $f$ satisfies most of the standard properties of probability distributions - in particular, it can be used to compute averages just as a classical distribution. However, it cannot in itself be regarded as a probability density because it can take negative values.

Alternatively, the QHD model can also be derived from the time-dependent Hartree equations. ${ }^{23}$ The latter derivation is useful for extending the approach to include densitydependent potentials that model exchange and correlation effects. In that case, the derivation should start from the timedependent Kohn-Sham equations. ${ }^{29}$ The Wigner distribution $f(x, v, t)$ and the Kohn-Sham wave functions $\phi_{k}(x, t)$ are related by the Wigner transformation:

$$
f(x, v, t)=\sum_{k=1}^{N} \frac{m}{2 \pi \hbar} p_{k} \int_{-\infty}^{+\infty} \phi_{k}^{*}\left(x+\frac{\lambda}{2}, t\right) \phi_{k}\left(x-\frac{\lambda}{2}, t\right) e^{i m v \lambda / \hbar} d \lambda,
$$

where the occupation probabilities $p_{k}$ are defined to describe a Fermi-Dirac distribution at zero temperature. Note that we restrict our discussion to one-dimensional (1D) geometry. This is appropriate for the study of thin films, where only variations along the direction $x$, normal to the film surface, are taken into account.

The QHD model involves the first two moments of the Wigner function, namely, the electron density $n(x, t)$ $=\int f(x, v, t) d v$ and the local mean velocity $u(x, t)$ $=\int f(x, v, t) v d v / n$. These quantities can also be expressed in terms of the Kohn-Sham wave functions:

$$
\begin{gathered}
n=\sum_{k=1}^{N} p_{k} n_{k}, \\
u=\sum_{k=1}^{N} p_{k} \frac{n_{k}}{n} u_{k},
\end{gathered}
$$

where

$$
\phi_{k}=\sqrt{n_{k}} \exp \left(i \frac{m}{\hbar} \int^{x} u_{k}\left(x^{\prime}, t\right) d x^{\prime}\right) .
$$

Finally, the QHD model reads as a set of a continuity and an Euler equation:

$$
\frac{\partial n}{\partial t}+\frac{\partial(n u)}{\partial x}=0
$$

$$
\frac{\partial u}{\partial t}+u \frac{\partial u}{\partial x}=\frac{e}{m} \frac{\partial V_{\mathrm{eff}}}{\partial x}+\frac{\hbar^{2}}{2 m^{2}} \frac{\partial}{\partial x}\left(\frac{\partial_{x}^{2} \sqrt{n}}{\sqrt{n}}\right)-\frac{1}{m n} \frac{\partial P}{\partial x},
$$

where $m$ and $e$ are the electron mass and absolute charge, respectively, and the effective potential $V_{\text {eff }}=V_{H}+V_{X}+V_{C}$ is given by the sum of the Hartree, exchange, and correlation contributions. In the adiabatic local-density approximation (ALDA), the exchange and correlation terms are functionals of the time-dependent electron density $n(x, t)$. Here, we shall use the functionals suggested by Brey et al. ${ }^{30}$ The Hartree potential obeys Poisson's equation 


$$
\frac{\partial^{2} V_{H}}{\partial x^{2}}=\frac{e}{\epsilon_{0}}\left[n(x, t)-n_{i}(x)\right],
$$

where $\epsilon_{0}$ is the vacuum dielectric constant and $n_{i}(x)$ is the ion density profile, which is supposed to be continuous and immobile (jellium approximation). The second term on the right-hand side of Eq. (6) is the so-called Bohm potential, which contains all the quantum effects that are present in the model.

The pressure term $P$ appearing in the Euler equation [Eq. (6)] must be related to the electron density $n$ via an equation of state in order to close the fluid system. ${ }^{23}$ Generally speaking, we can take a polytropic relation

$$
P=P_{0}\left(\frac{n}{n_{0}}\right)^{\gamma}
$$

where $P_{0}=(2 / 5) n_{0} E_{F}$ is the pressure of a quantum electron gas at zero temperature (Fermi pressure), $E_{F}$ is the Fermi energy, $n_{0}$ the equilibrium density, and $\gamma=5 / 3$. Note that we use the three-dimensional (3D) value for the polytropic exponent. This reflects the fact that the equilibrium is fully $3 \mathrm{D}$, whereas the nonequilibrium dynamics only affects the direction normal to the film surfaces.

It is important to stress that most hydrodynamic models developed for metal clusters and nanoparticles ${ }^{7,17,18}$ do not include the Bohm potential in the Euler equation (quantum effects are only to be found in the equation of state, that is, the relation between the pressure and the density). However, a simple estimate shows that, for nanometric objects, the Bohm potential $V_{\text {Bohm }}$ is of the same order of magnitude as the Fermi pressure $P_{0}$ :

$$
V_{\mathrm{Bohm}}=\frac{\hbar^{2}}{2 m}\left(\frac{\partial_{x}^{2} \sqrt{n}}{\sqrt{n}}\right) \sim \frac{\hbar^{2}}{m \lambda^{2}}, \quad P_{0} / n_{0} \sim E_{F},
$$

where $\lambda$ is the typical length scale of the electron density. Assuming that $\lambda \simeq L_{F}$, one obtains that $V_{\mathrm{Bohm}} / E_{F}$ $\sim\left(\hbar \omega_{\mathrm{pe}} / E_{F}\right)^{2} \sim 1$, indicating that both terms have a similar impact and should thus both be retained in the model.

In the forthcoming simulations, time is normalized in units of the inverse plasmon frequency $\omega_{\mathrm{pe}}=\left(e^{2} n_{0} / m \epsilon_{0}\right)^{1 / 2}$, velocity in units of the Fermi speed $v_{F}$, and length in units of $L_{F}=v_{F} / \omega_{\text {pe }}$. In addition, particle densities are normalized to the ion density of the bulk metal $n_{0}=3 /\left(4 \pi r_{s}^{3}\right)$, where $r_{s}$ is the Wigner-Seitz radius. With these normalizations, the exchange and correlation potentials read as ${ }^{30}$

$$
\begin{gathered}
V_{X}=0.985 \frac{H^{2} \kappa}{4 \pi} n^{1 / 3}, \\
V_{C}=0.03349 \frac{H^{4} \kappa^{3}}{(4 \pi)^{2}} \ln \left(1+\frac{4 \pi \times 18.376}{H^{2} \kappa^{2}} n^{1 / 3}\right),
\end{gathered}
$$

where $H=\hbar \omega_{\mathrm{pe}} / m v_{F}^{2}$ is the normalized Planck constant and $\kappa=\left(3 \pi^{2}\right)^{2 / 3}$. Here, we will concentrate on sodium films, for which $r_{s} / a_{0}=4\left(a_{0}=0.529 \AA\right.$ is the Bohr radius $)$, so that we have $L_{F}=1.2 \AA, E_{F}=3.1 \mathrm{eV}, \omega_{\mathrm{pe}}^{-1}=0.1$ fs, and $H=0.9405$.
An important feature of the QHD equations [Eqs. (5) and (6)] is that they can be rewritten in the form of a single nonlinear Schrödinger equation by making use of a Madelung transformation

$$
\Psi(x, t)=\sqrt{n(x, t)} \exp [i S(x, t) / \hbar],
$$

where $\Psi$ is an effective wave function, $|\Psi|^{2}=n$, and $S(x, t)$ is such that $m u(x, t)=\partial_{x} S(x, t)$. We then obtain that $\Psi(x, t)$ satisfies the following nonlinear Schrödinger equation, in normalized units:

$$
i H \frac{\partial \Psi}{\partial t}=-\frac{H^{2}}{2} \frac{\partial^{2} \Psi}{\partial x^{2}}-V_{\text {eff }} \Psi+W_{F} \Psi .
$$

The "Fermi" potential $W_{F}$ originates from the Fermi pressure $P$ :

$$
W_{F}=\int \frac{1}{n} \frac{d P}{d n} d n,
$$

and, for a polytropic equation of state [Eq. (8)], it takes the following form:

$$
W_{F}=\frac{\gamma}{5(\gamma-1)}|\Psi|^{2(\gamma-1)} .
$$

Finally, the normalized Poisson's equation reads as

$$
\frac{\partial^{2} V_{H}}{\partial x^{2}}=|\Psi|^{2}-n_{i} / n_{0}
$$

Written in this form, the QHD model consists of the nonlinear Schrödinger equation [Eq. (13)], together with the definitions of the exchange, correlation, and Fermi potentials-Eqs. (10), (11), and (14), respectively-and Poisson's equation [Eq. (16)]. This form of the QHD model is particularly well suited for numerical simulations because it eliminates the "difficult" nonlinearities represented by the convective term $\left(u \partial_{x} u\right)$ and the Bohm potential in the Euler Eq. (6).

\section{GROUND STATE}

Before attacking the study of the electron dynamics, we investigate the ground state of the QHD model. In order to compute the ground state, a useful numerical technique relies on the "imaginary time" approach. This consists in replacing $t$ with $-i H t$ in Eq. (13), thus transforming the Schrödinger equation into a diffusion equation with nonlinear sources

$$
\frac{\partial \Psi}{\partial t}=\frac{H^{2}}{2} \frac{\partial^{2} \Psi}{\partial x^{2}}+V_{\mathrm{eff}} \Psi-\frac{\gamma}{5(\gamma-1)}|\Psi|^{2(\gamma-1)} \Psi .
$$

The long-time solution of this diffusion equation (coupled to Poisson's equation) will generally converge to a stationary state that coincides with the ground state of the original Schrödinger equation. However, as the diffusion equation does not conserve the $L^{2}$ norm of the wave function $\left(\int|\Psi|^{2} d x\right)$, it is necessary to renormalize $\Psi$ at every time step. This renormalization effectively sets the value of the chemical potential $\mu$ (energy eigenvalue of the ground state): 

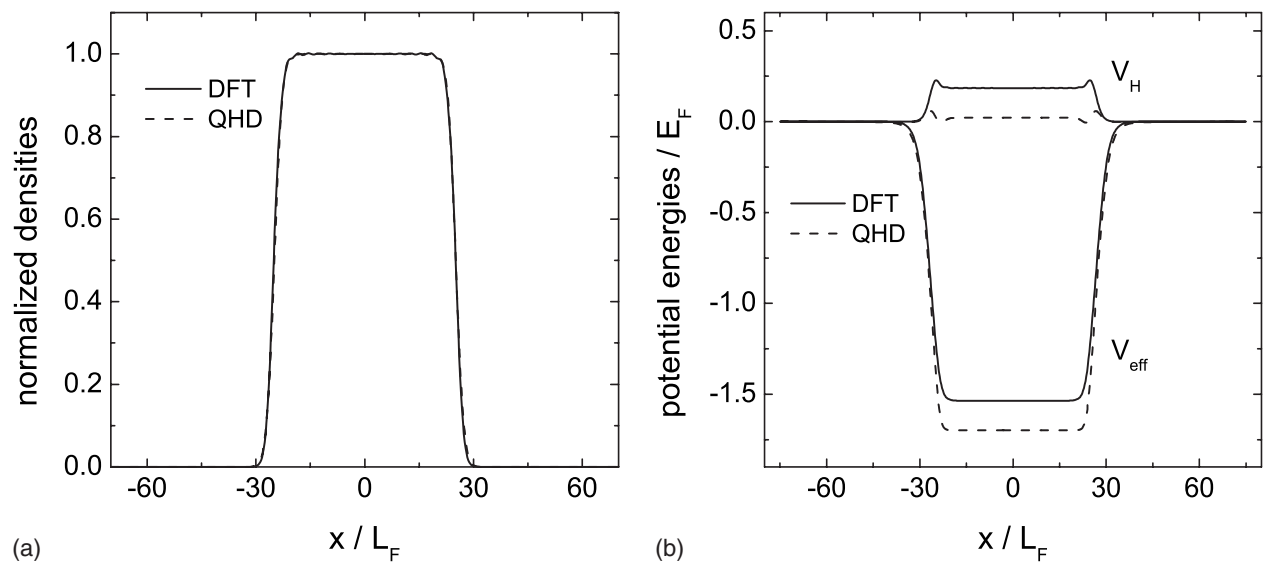

FIG. 1. Comparison between the ground states computed from the DFT equations (solid line) and with the QHD approach (dashed line) for a film of thickness $L=50 L_{F}$ and $\sigma=L_{F}$. (a) Electron density normalized to the background density $n_{0}$ (b) Profile of the Hartree potential $\left(V_{H}\right)$ and effective potential $\left(V_{\text {eff }}\right)$ normalized to the Fermi energy.

$$
-\frac{H^{2}}{2} \frac{\partial^{2} \Psi}{\partial x^{2}}-V_{\text {eff }} \Psi+\frac{\gamma}{5(\gamma-1)}|\Psi|^{2(\gamma-1)} \Psi=\mu \Psi .
$$

To compute the stationary solution, we solve Eq. (17) using an implicit numerical scheme with centered differences to evaluate the spatial derivatives. The order of the timestepping scheme is not crucial as we are only interested in the time-asymptotic solution. In contrast, its stability is mandatory, and this is why we use an implicit technique. The numerical resolution is stopped when the quantities of interest (kinetic and potential energies for example) converge to a constant value, within some given accuracy.

We assume that the ions form a motionless neutralizing background represented by a positive and inhomogeneous charge density

$$
n_{i}(x)=\frac{1}{1+\exp [(|x|-L / 2) / \sigma]},
$$

where $\sigma$ is a diffuseness parameter and $L$ is the thickness of the slab. Homogeneous Dirichlet boundary conditions are used at the boundaries of the computational box $\left[-L_{\max }, L_{\max }\right]$, with $L_{\max } \gg L$. Initially, the electron density has the same form as the ion density but with slightly larger $\sigma$. This is what we expect the ground state to look like, and this choice allows us to minimize the number of iterations necessary to converge.

The results of the QHD model are compared to those obtained using a DFT solver for the same physical system. In the Kohn-Sham formulation of DFT, the ground-state density $n(x)$ of an $N$-electron system is written, in terms of the single-particle orbitals $\phi_{k}$ and energies $\varepsilon_{k}$, as

$$
n(x)=\sum_{k=1}^{N} p_{k}\left|\phi_{k}(x)\right|^{2}
$$

These orbitals and energies obey the Schrödinger equations $(k=1 \ldots N)$

$$
\left[-\frac{\hbar^{2}}{2 m} \frac{\partial^{2}}{\partial x^{2}}+V_{\mathrm{eff}}(x)\right] \phi_{k}(x)=\varepsilon_{k} \phi_{k}(x)
$$

and the last occupied energy level defines the Fermi energy. The Kohn-Sham equations [Eqs. (21)] are solved numerically using a standard finite-difference iterative method. ${ }^{31}$

We consider two test cases, both with film thickness $L=50 L_{F}$ but with different values of the diffuseness parameter, $\sigma=L_{F}$ and $\sigma=2 L_{F}$. The size of the computational box is equal to $2 L_{\max }=150 L_{F}$ with $N_{x}=1500$ mesh points.

In Fig. 1, the electron densities and the Hartree and effective potentials are plotted as functions of the normalized spatial coordinate for the case $\sigma=L_{F}$. The electron density is basically identical for both models, although some discrepancy remains for the computed potentials. A better accordance on the potentials is obtained for $\sigma=2 L_{F}$ (Fig. 2). This is in line with the underlying assumptions of the QHD model, which requires that all quantities vary on distances not shorter than the screening length $L_{F}$.

This fact appears clearly in Fig. 3, where we plot the Thomas-Fermi energy and the effective potential energy against $\sigma$. In our normalized units, these energies are defined as follows:

$$
E_{T F}=\frac{2}{5(\gamma-1)} \int|\Psi|^{2 \gamma} d x, \quad E_{\text {eff }}=\int V_{\text {eff }}(x)|\Psi|^{2} d x,
$$

with $\gamma=5 / 3$. The Thomas-Fermi energy yields good results for all values of $\sigma$. This is not surprising as $E_{T F}$ is simply a functional of the density, and we have seen that the density computed with the QHD model agrees well with the DFT result irrespective of $\sigma$ (as far as it is not too small). The test on the effective potential energy is more stringent. We spanned values of $\sigma$ going from 0.5 to $2.5 L_{F}$ (larger values are not physically meaningful) and observed that the DFT and QHD results (slowly) converge for large $\sigma$.

In summary, the QHD approach yields satisfactory results for the computation of the ground state, provided that the spatial profiles do not vary too abruptly. This limitation is 

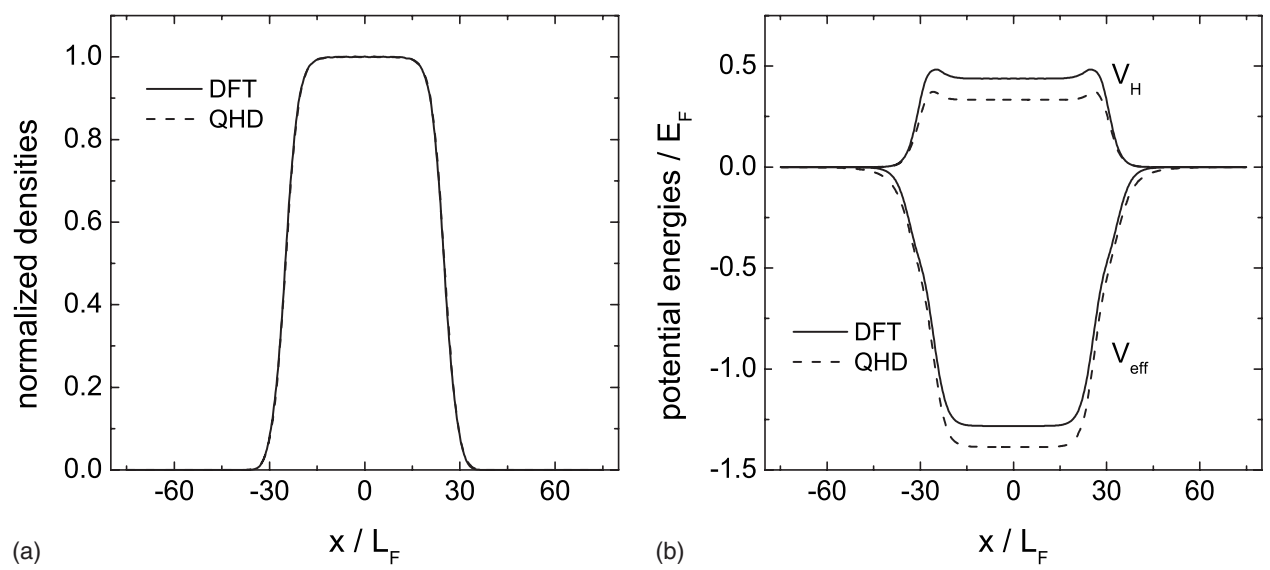

FIG. 2. Same as Fig. 1 for $\sigma=2 L_{F}$.

inherent to most hydrodynamic models (both classical and quantum). However, even for rather steep variations in the ion density $\left(\sigma \simeq 0.5 L_{F}\right)$, the electron density profile is still accurate and the relative error on the effective potential energy is only around $10 \%$.

\section{DYNAMICS}

Recent semiclassical simulations ${ }^{19-21}$ have revealed an interesting nonlinear regime in the electron dynamics in a thin metal film. In these studies, the electrons were initially prepared in a Fermi-Dirac equilibrium at finite (but small) temperature. They were subsequently excited by imposing a constant velocity shift $\delta v$ to the initial distribution. This scenario is appropriate when no linear momentum is transferred parallel to the plane of the surface (i.e., $q_{\|}=0$ ) and is relevant to the excitation of the film with optical pulses. ${ }^{32}$ For $q_{\|}=0$, only longitudinal modes (plasmon with $\omega \simeq \omega_{\text {pe }}$ ) can be excited. It was observed that after the initial absorption of the perturbation energy (which was rapidly transformed into thermal energy), a regime of low-frequency oscillations appeared. The period of the oscillations corresponded to electrons traveling at the Fermi velocity and bouncing back and forth on the film surfaces.

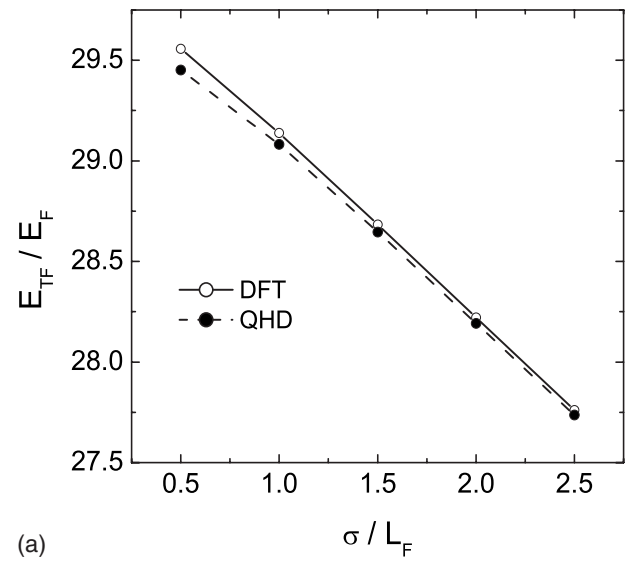

The above simulations were carried out within the framework of the semiclassical Vlasov-Poisson equations, thus neglecting quantum-mechanical effects. The Vlasov equation describes the evolution of a classical probability distribution in phase space $f(x, v, t)$ :

$$
\frac{\partial f}{\partial t}+v \frac{\partial f}{\partial x}+\frac{e}{m} \frac{\partial V_{H}}{\partial x} \frac{\partial f}{\partial v}=0
$$

where the Hartree potential $V_{H}$ obeys Poisson's equation [Eq. (7)]. The Vlasov equation can be obtained as the classical limit of the Wigner or Kohn-Sham equations.

Our purpose here is to investigate the same physical problem with the QHD model described in Sec. III. Note that the Vlasov and QHD approaches are in some sense complementary. The Vlasov approach is microscopic (at the mean-field level) but classical, whereas the QHD approach is macroscopic but includes quantum effects.

The electrons are initially prepared in the ground state, computed as described in Sec. III. In order to excite the dynamics, the initial state is suddenly accelerated by a velocity $\delta v$ (normalized units are used):

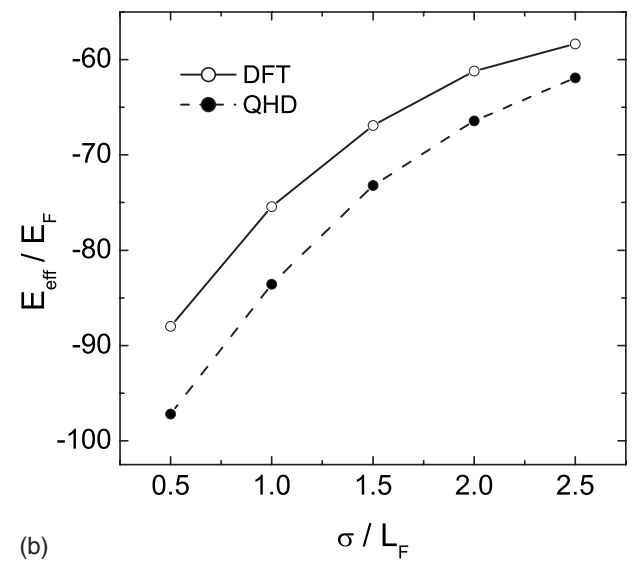

FIG. 3. Ground-state properties: Thomas-Fermi energy (a) and effective potential energy (b) against $\sigma / L_{F}$ computed using the DFT equations and the QHD approach for a film of thickness $L=50 L_{F}$. Energies are normalized to the Fermi energy. 


$$
\Psi\left(x, t=0^{+}\right)=\Psi\left(x, t=0^{-}\right) \exp \left(-i \frac{\delta v}{H} x\right) .
$$

This excitation corresponds to the one used for the Vlasov model, i.e., a uniform shift $\delta v$ in velocity space.

A time-splitting technique ${ }^{33}$ has been employed to integrate numerically the time-dependent nonlinear Schrödinger equation [Eq. (13)]. Let us write the Schrödinger equation as

$$
i H \frac{\partial \Psi}{\partial t}=(K+V) \Psi,
$$

where $K$ is the kinetic energy operator and $V=W_{F}-V_{\text {eff }}$ is the total potential energy. The Hamiltonian is split into these two parts and each is treated separately. For the potential part, the solution is trivial

$$
\Psi^{n+1}=\Psi^{n} \exp (-i V \Delta t / H),
$$

where $\Psi^{n} \equiv \Psi(n \Delta t)$ and $\Delta t$ is the time step. For the kinetic part, we use a Crank-Nicolson scheme, which is exact to second order in $\Delta t$,

$$
i H \frac{\Psi^{n+1}-\Psi^{n}}{\Delta t}=\frac{1}{2}(K \Psi)^{n+1}+\frac{1}{2}(K \Psi) \Psi^{n} .
$$

The kinetic operator $K$ is approximated with the standard centered-differences formula. The time evolution is obtained by subsequently applying the potential and kinetic steps described above. Poisson's equation is solved just before the potential step (notice that the kinetic step does not alter the spatial density and therefore the potential $V$ ). The resulting numerical scheme is unconditionally stable and second order accurate in both space and time. Another crucial property of the scheme is that it conserves exactly the $L^{2}$ norm $\int|\Psi|^{2} d x$.

We are interested in the evolution of certain energy quantities. The total energy of the electron population (which is a conserved quantity for the QHD model) can be written as the sum of several terms: $E_{\mathrm{tot}}=E_{\mathrm{kin}}+E_{T F}+E_{\mathrm{pot}}+E_{X C}$, where

$$
\begin{aligned}
& E_{\text {kin }}=\frac{H^{2}}{2} \int\left|\frac{\partial \Psi}{\partial x}\right|^{2} d x, \\
& E_{T F}=\frac{2}{5(\gamma-1)} \int n^{\gamma} d x, \\
& E_{\mathrm{pot}}=\frac{1}{2} \int\left(n_{i}-n\right) V_{H} d x .
\end{aligned}
$$

Here, $E_{T F}$ is the Thomas-Fermi energy and $E_{\text {pot }}$ is the electrostatic potential energy. The exchange-correlation energy $E_{X C}$ is a complicated functional of the electron density that we do not need to write explicitly. The kinetic energy $E_{\text {kin }}$ can be split into the energy of the center of mass,

$$
E_{\mathrm{cm}}=\frac{1}{2} \int \frac{j^{2}}{n} d x=\frac{1}{2} \int n u^{2} d x,
$$

and a residual energy, which we refer to as the "thermal energy," $E_{\mathrm{th}}=E_{\mathrm{kin}}-E_{\mathrm{cm}}$. The current is defined (in dimensionless units) as $j=H \operatorname{Im}\left(\Psi^{*} \partial_{x} \Psi\right)=n u$, where $\Psi^{*}$ denotes the complex conjugate of $\Psi$.
Here, some explanation is in order as we have so far supposed that the electron system is at zero temperature. The "thermal energy" represents an internal kinetic energy that goes beyond both the Thomas-Fermi energy (i.e., the kinetic energy of a zero-temperature Fermi gas) and the kinetic energy due to the motion of the center of mass. Basically, the thermal energy is due to the uncertainty in velocity arising from Heisenberg's relations. This is of course a purely quantum effect-for an equivalent classical model the thermal energy would be strictly equal to zero. Thus, the QHD model leaves open the possibility to account for an increase in the electron thermal energy and therefore the electron temperature.

It is easy to estimate the thermal energy of the ground state, for which $E_{\mathrm{cm}}=0$ and thus $E_{\mathrm{th}}=E_{\mathrm{kin}}$. The kinetic energy is zero inside the film, where the wave function is spatially uniform. At the boundaries, the typical velocity dispersion is given by the uncertainty principle $\Delta v=\hbar /(m \sigma)$, with $\sigma$ $\simeq L_{F}$. Then, the thermal energy can be estimated as $E_{\mathrm{th}}$ $\sim m \Delta v^{2}=\left(\hbar \omega_{\mathrm{pe}}\right)^{2} / m v_{F}^{2}$. In our normalized units, we obtain that $E_{\mathrm{th}} / m v_{F}^{2} \sim H^{2}$. The Thomas-Fermi energy can be computed from Eq. (29) by assuming a flat density profile for $|x| \leq L / 2$ and $n=0$ elsewhere. This yields, again in normalized units, $E_{T F}=\frac{2}{5(\gamma-1)} \frac{L}{L_{F}}$, independently of $H$. Therefore, for large enough films, the Thomas-Fermi energy always exceeds the thermal energy in the ground state. Of course, this need not be the case out of equilibrium, as we shall see in the forthcoming simulations.

We now present the simulation results obtained with the QHD model for a film of thickness $L=50 L_{F}$ or $L=100 L_{F}$, diffuseness parameter $\sigma=2 L_{F}$, and $\delta v=0.22 v_{F}$. The numerical resolution is as follows: $\Delta x / L_{F}=0.1$ and $\omega_{\mathrm{pe}} \Delta t=5$ $\times 10^{-3}$.

Before looking at the various energy terms, we study the evolution of the electric dipole $d(t)$, defined as

$$
d(t)=\frac{\int x|\Psi(x, t)|^{2} d x}{\int|\Psi(x, t)|^{2} d x} .
$$

In Fig. 4, we plot the time history of the dipole together with its frequency spectrum (normalized to its maximum value) for different values of $H$, ranging from $H=0.9405$ (which corresponds to sodium films with $\left.r_{s} / a_{0}=4\right)$ to $H=0.1$, and film thickness $L=50 L_{F} \simeq 60 \AA$. In all cases, the spectrum is peaked around $\omega \simeq 0.8 \omega_{\text {pe }}$, i.e., slightly smaller than the plasmon frequency. As explained in Ref. 20, this is a spurious effect due to the finite size of the computational box. The smaller peaks appearing at low frequency are a manifestation of individual electron excitations in the confining potential.

It is interesting to note that, although the QHD equations are not, in principle, capable of modeling effects like Landau damping, a significant decrease in the dipole is still observed. Like Landau damping, the electric-dipole damping observed in Fig. 4 involves a transfer of energy from the center of mass to the internal degrees of freedom of the electron gas. From the logarithmic plot of the dipole, the damping rate turns out to be $\gamma \simeq 0.055 \omega_{\text {pe }}$ for all values of $H$. The expo- 


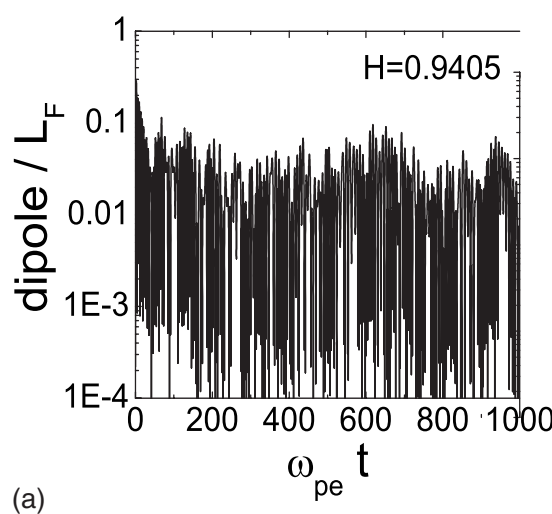

(a)
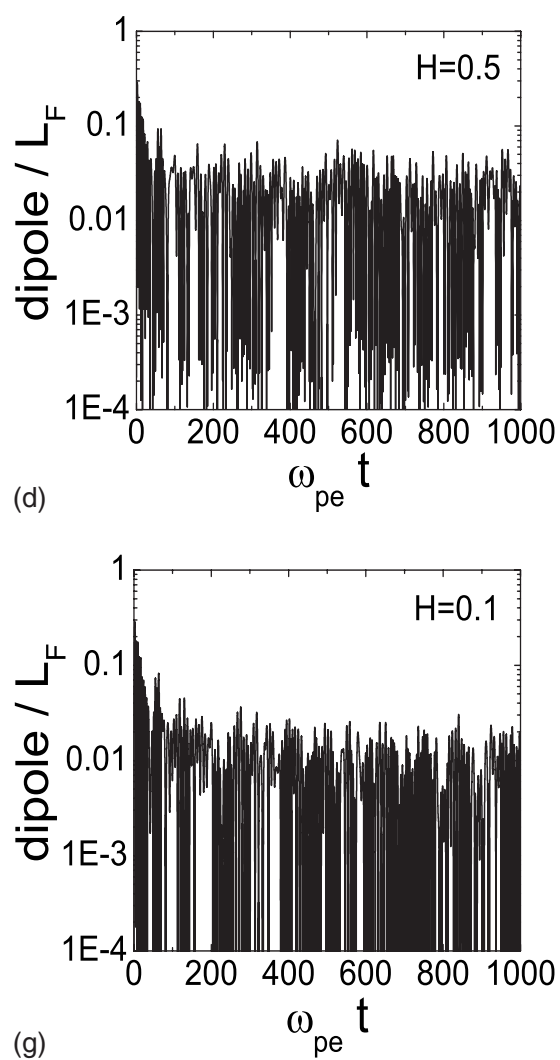
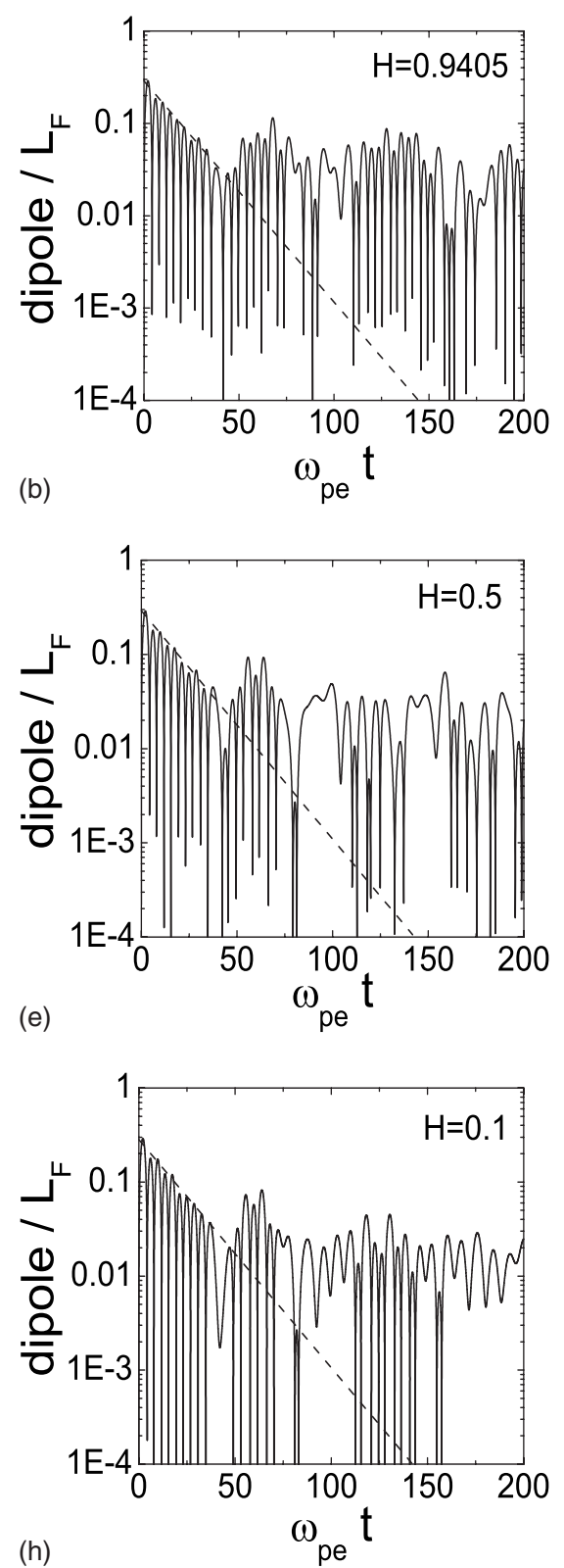
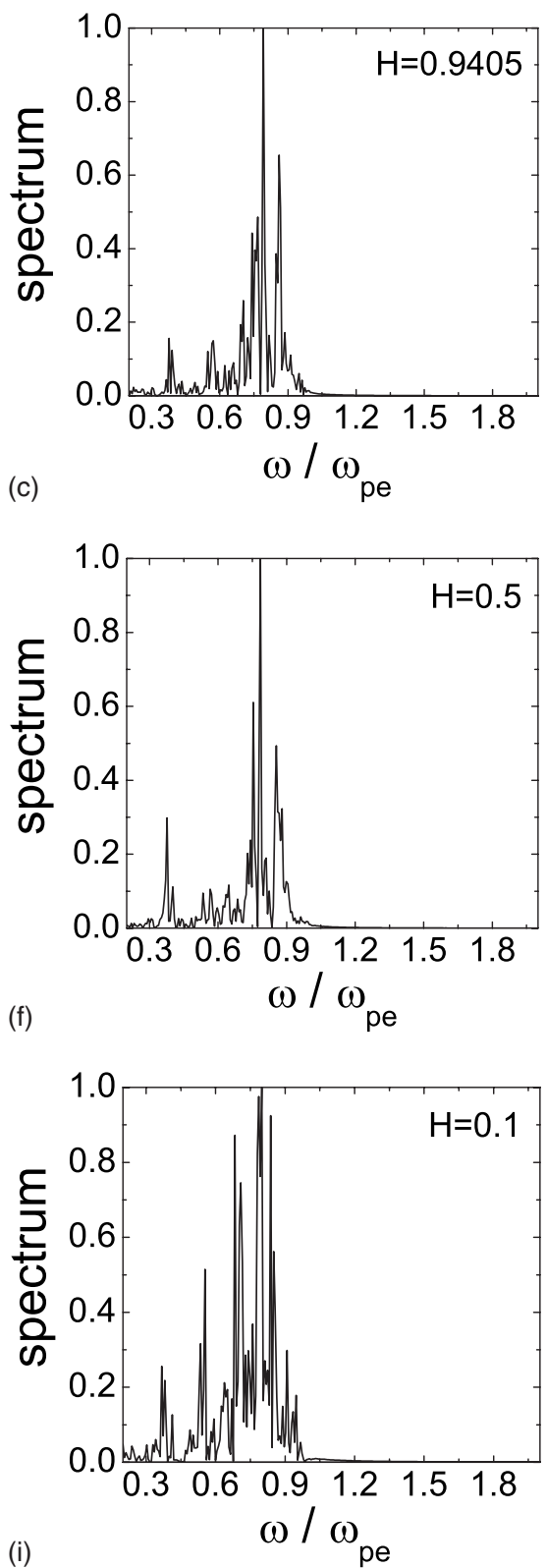

FIG. 4. QHD model. Electric dipole for $L=50 L_{F}$ and $\sigma=2 L_{F}$. Left column: Full time history on a logarithmic scale; middle column: Zoom for short times (the dashed straight line is an exponential with damping rate $\gamma=0.055 \omega_{\mathrm{pe}}$ ); right column: Frequency spectrum. From top to bottom: $H=0.9405, H=0.5, H=0.1$.

nential damping stops after $50 \omega_{\mathrm{pe}}^{-1}$, which corresponds to the time required for electrons located at the Fermi surface to travel across the film. The long-time amplitude of the dipole saturates at a level that decreases with decreasing $H$.

Figure 5 shows the electric dipole obtained from the Vlasov-Poisson simulations. The general behavior is quite similar to that observed for the QHD results. The initial damping rate is smaller $\left(\gamma_{\mathrm{Vlas}}=0.035 \omega_{\mathrm{pe}}\right)$, but the long-time decay is more pronounced for the Vlasov case. Indeed, at $\omega_{\mathrm{pe}} t=1000$ the dipole is smaller than $10^{-2}$ for the Vlasov result, whereas it is larger than $10^{-2}$ for the QHD result, even for $H=0.1$. Nevertheless, the QHD results for the long-time value of the dipole show a decreasing trend with $H$ so that the Vlasov result may well be attained in the limit $H \rightarrow 0$. The classical spectrum is also peaked at $\omega \simeq 0.8 \omega_{\mathrm{pe}}$, although it displays a less complex structure than its quantum counterpart.

Figure $6\left(L=50 L_{F}\right)$ and Fig. $7\left(L=100 L_{F}\right)$ show the evolution of $E_{\mathrm{pot}}, E_{\mathrm{cm}}$, and $E_{\mathrm{th}}$ in units of the Fermi energy for several values of $H$. First, we note that all these quantities obey a trivial scaling law with the system size; i.e., they are twice as large for the film with thickness $L=100 L_{F}$. We thus concentrate our discussion on the case $L=100 L_{F}$.

In the semiclassical limit $(H=0.1)$, the overall behavior of the energies is very similar to that observed in the VlasovPoisson simulations (Fig. 8). In particular: (i) The electrostatic potential energy saturates at a level very close to the classical result. (ii) The thermal energy increases rapidly from an initially small value (as explained above, the ground-state thermal energy decreases with $H$, which is vis- 

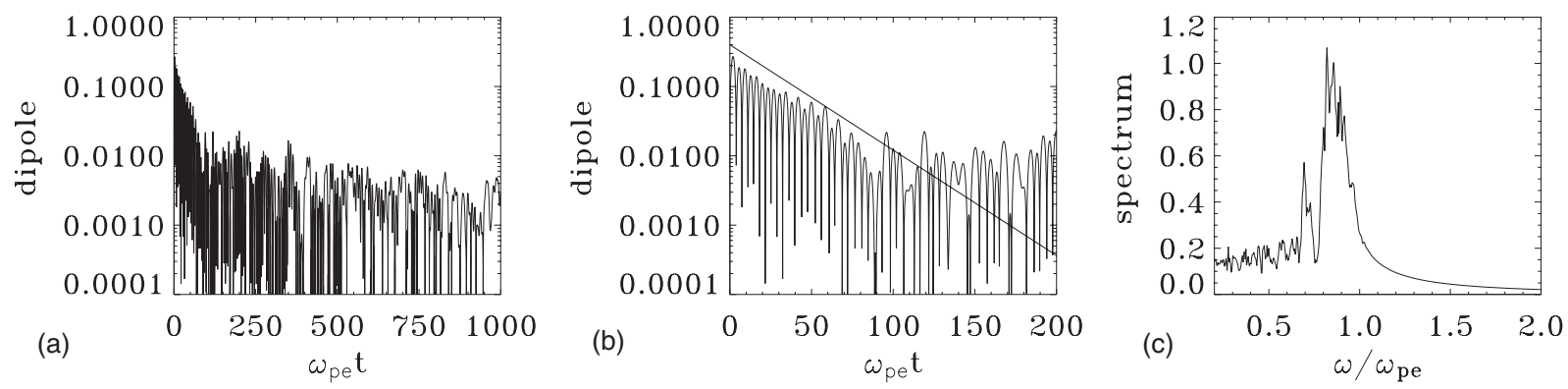

FIG. 5. Electric dipole from the Vlasov simulations for $L=50 L_{F}$ and $\sigma=2 L_{F}$. (a) Full time history on a logarithmic scale. (b) Zoom for short times. (c) Frequency spectrum. The straight line is an exponential with damping rate $\gamma_{\mathrm{Vlas}}=0.035 \omega_{\text {pe }}$.

ible on the figure), then saturates at a value close to $E_{F}$ for the case with $L=100 L_{F}$ (Fig. 7). (iii) A regime of slow nonlinear oscillations is clearly visible for $H=0.1$, particularly on the potential energy (bottom left frames in Figs. 6 and 7). For the Vlasov results (Fig. 8), the regime of slow oscilla- tions was interpreted as the result of electrons traveling ballistically through the film at the Fermi speed and bouncing back and forth on the surfaces. ${ }^{19,20}$ The period of the oscillations is roughly equal to $L / v_{F}$, although not exactly. The slight discrepancy comes from the fact that the actual thick-
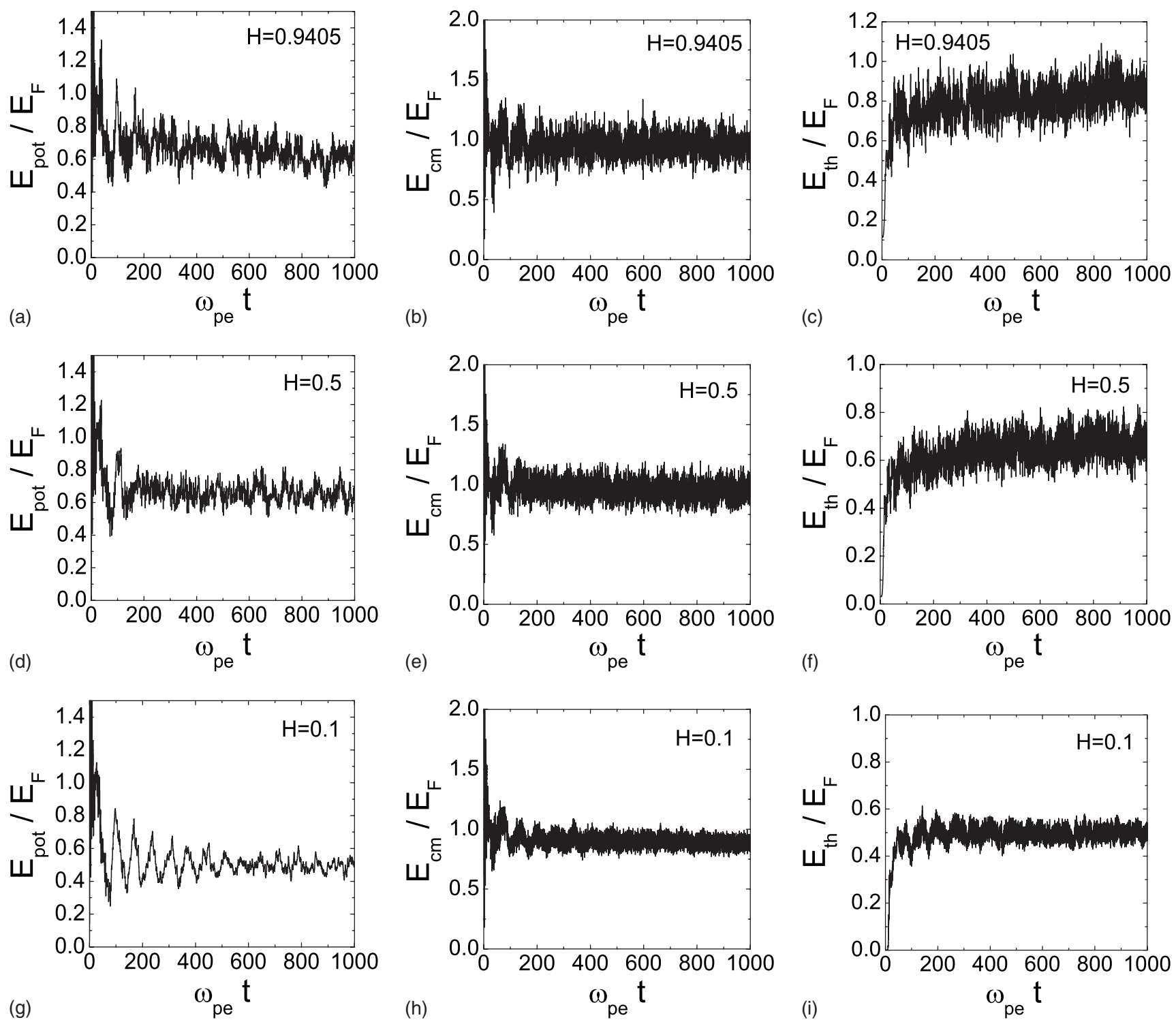

FIG. 6. QHD model. Time evolution of the potential energy (left column), center-of-mass energy (middle column), and thermal energy (right column) for $L=50 L_{F}, \sigma=2 L_{F}$. From top to bottom: $H=0.9405, H=0.5, H=0.1$. Energies are expressed in units of the Fermi energy. 

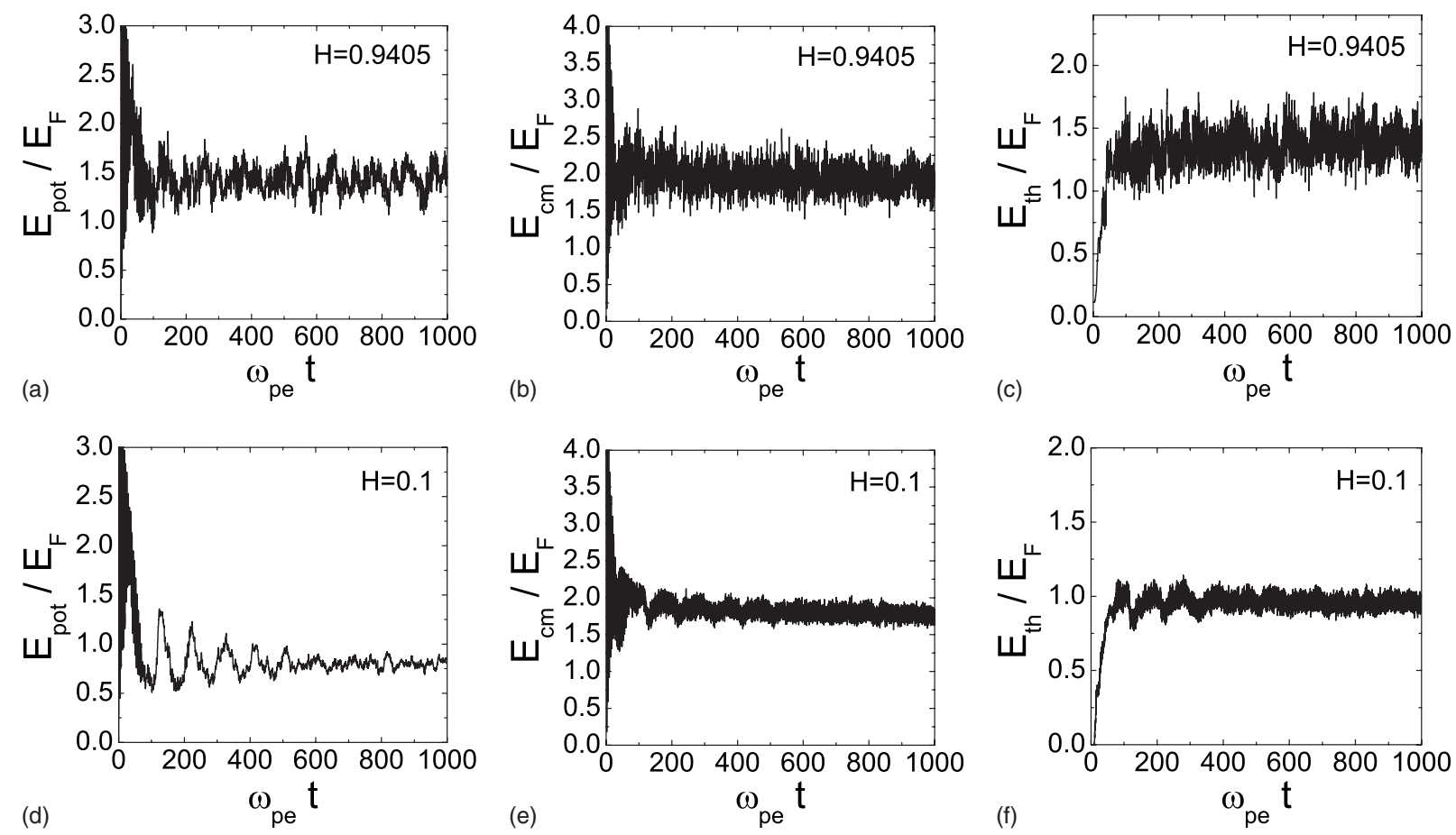

FIG. 7. QHD model. Time evolution of the potential energy (left column), center-of-mass energy (middle column), and thermal energy (right column) for $L=100 L_{F}, \sigma=2 L_{F}$. Top panels: $H=0.9405$; bottom panels: $H=0.1$. Energies are expressed in units of $E_{F}$.

ness of the slab is bigger than its nominal value $L$ (see Fig. 2 ). In addition, the relatively large value of $\delta v$ used here means that velocities higher than $v_{F}$ are excited, thus reducing the ballistic period.

We also observe that the sum of the kinetic, center of mass, and potential energies at the end of the run is roughly equal to $5 E_{F}$ for the Vlasov results but only to $4 E_{F}$ for the QHD case. The reason is that for the Vlasov case, the Thomas-Fermi energy hardly varies at all during the entire run so that the sum $E_{\mathrm{cm}}+E_{\mathrm{pot}}+E_{\mathrm{th}}$ is approximately constant. However, for the QHD case the Thomas-Fermi energy slightly increases over the run (just above $1 \%$ of its initial value), which is not much in relative terms, but as $E_{T F}$ is large compared to the other energy terms, it is enough to account for the observed discrepancy (in the above discussion, we have ignored exchange and correlations as we are considering the case $H=0.1$ ).

The variation in the different energy terms over the entire run is shown in Table I (Vlasov) and Table II (QHD) for the film of thickness $L=100 L_{F}$. The final values represent an average in the range $\omega_{\mathrm{pe}} t=900-1000$. In the Vlasov case, the initial excitation energy (center of mass) is transferred almost entirely to thermal energy $(85 \%)$ and electrostatic potential

TABLE I. Initial and final values of the energies (in units of $\left.E_{F}\right)$ for the Vlasov simulations with $L=100 L_{F}$.

\begin{tabular}{lccccc}
\hline \hline & $E_{\mathrm{cm}}$ & $E_{\mathrm{th}}$ & $E_{\mathrm{pot}}$ & $E_{T F}$ & Total \\
\hline Initial & 4.84 & 0.01 & 0.03 & 58.11 & 62.99 \\
Final & 0.01 & 4.14 & 0.74 & 58.05 & 62.94 \\
Variation & -4.83 & 4.13 & 0.71 & -0.05 & -0.04 \\
\hline \hline
\end{tabular}

energy $(15 \%)$. For the quantum case, the situation is more complex. Of the initial excitation energy, only about $20 \%$ goes into thermal energy, whereas $37 \%$ remains as center-ofmass energy (i.e., coherent macroscopic oscillations), $16 \%$ is converted into potential energy (the same amount as in the Vlasov case), and about $15 \%$ is transferred to the ThomasFermi energy.

The observed incomplete damping of the center-of-mass energy clearly represents a deficiency of the QHD approach compared to a phase-space description and is due to the absence of Landau damping in the hydrodynamic model. A possible solution could be to add a small artificial friction term $-\eta u$ on the right-hand side of the Euler equation [Eq. (6)], although the choice of the damping rate $\eta$ would remain problematic. This procedure would introduce a current dependency in the effective potential. This avenue to model irreversible effects has been explored by Vignale and Kohn ${ }^{34}$ (see also more recent papers citing this one), who proposed a generalization of DFT based on the current density functional. Their general approach could possibly be adapted to
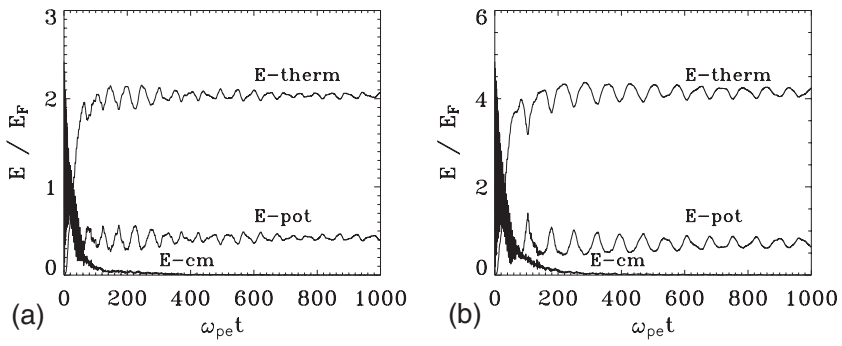

FIG. 8. Time evolution of the potential, center-of-mass and thermal energies for the Vlasov-Poisson model. (a) $L=50 L_{F}$. (b) $L$ $=100 L_{F}$. For both cases, $\sigma=2 L_{F}$. 
TABLE II. Initial and final values of the energies (in units of $E_{F}$ ) for the QHD simulations $(H=0.1)$ with $L=100 L_{F}$.

\begin{tabular}{lccccc}
\hline \hline & $E_{\mathrm{cm}}$ & $E_{\mathrm{th}}$ & $E_{\mathrm{pot}}$ & $E_{T F}$ & Total \\
\hline Initial & 4.82 & 0.00 & 0.03 & 58.11 & 62.96 \\
Final & 1.77 & 0.96 & 0.80 & 58.84 & 62.37 \\
Variation & -3.05 & 0.96 & 0.77 & 0.73 & -0.59 \\
\hline \hline
\end{tabular}

the QHD model illustrated in the present work.

\section{CONCLUSION}

In this paper, we investigated in detail the ultrafast electron dynamics in a thin metal film using a quantum hydrodynamic model. The results were compared to ground-state calculations using the Kohn-Sham equations of densityfunctional theory, whereas the nonequilibrium dynamics was compared to recent results obtained with a semiclassical model. In contrast to previous investigations, the full nonlinear dynamics was analyzed, going well beyond the simple electric-dipole response.

The overall results show that the QHD model is capable of capturing the essential features of the electron dynamics, in particular: (i) the dipole response at the plasmon frequency and its decay, (ii) the initial heating of the electron gas to temperatures significantly higher than the equilibrium, and (iii) the subsequent regime of ballistic nonlinear oscillations with a period close to the time of flight between the film's surfaces. It is particularly intriguing that an increase in the thermal energy can actually be observed in a model that is nominally valid at zero temperature.

The limitations of the QHD model, which were expected from its theoretical underpinning, are essentially twofold. First, like for other fluid models, wavelengths shorter than the Thomas-Fermi screening length $L_{F}$ are not treated correctly. This limitation affects the calculation of the ground state. Second, the center-of-mass contribution to the kinetic energy does not decay away by Landau damping because the latter is absent from the QHD model. However, we point out that Landau damping was probably overestimated in the semiclassical simulations ${ }^{19,20}$ because the classical energy spectrum is by definition continuous. Landau damping is basically a phase-mixing effect that arises from many different states oscillating at slightly different frequencies and is thus amplified in the semiclassical regime, where the number of states is effectively infinite. When the spectrum is discrete and the number of states is finite, beatings and revivals can occur, thus reducing the efficiency of Landau damping.

In addition, first-order moments such as the electric dipole are indeed damped in the QHD evolution. The damping rate and the long-time saturation level are quantitatively similar to those observed in the Vlasov simulations. Thus, it seems that significant discrepancies arise only when computing second-order moments, i.e., the various energy terms.

All things considered, the incomplete damping of the center-of-mass energy does constitute a shortcoming of the present model. Recent theoretical results on a generalization of DFT that involves the electron current as well as the density could provide a means to model irreversible phenomena in the context of quantum hydrodynamics. At the simplest level, a friction term in the Euler equation would guarantee the damping of the center-of-mass energy, although the damping rate would have to be fixed ad hoc. Another possibility could be to develop a two-population model, whereby the zero-temperature component of the electron gas coexists with a thermal population, which might be treated semiclassically. The thermal population would thus provide the necessary amount of damping. These generalizations would considerably enlarge the range of applicability of the quantum hydrodynamic model described in this work.
*Giovanni.Manfredi@ipcms.u-strasbg.fr

${ }^{1}$ G. L. Eesley, Phys. Rev. Lett. 51, 2140 (1983).

${ }^{2}$ S. D. Brorson, J. G. Fujimoto, and E. P. Ippen, Phys. Rev. Lett. 59, 1962 (1987).

${ }^{3}$ C. Suárez, W. E. Bron, and T. Juhasz, Phys. Rev. Lett. 75, 4536 (1995).

${ }^{4}$ R. H. M. Groeneveld, R. Sprik, and A. Lagendijk, Phys. Rev. B 51, 11433 (1995).

${ }^{5}$ C.-K. Sun, F. Vallée, L. H. Acioli, E. P. Ippen, and J. G. Fujimoto, Phys. Rev. B 50, 15337 (1994).

${ }^{6}$ J.-Y. Bigot, V. Halté, J.-C. Merle, and A. Daunois, Chem. Phys. 251, 181 (2000).

${ }^{7}$ F. Calvayrac, P.-G. Reinhard, E. Suraud, and C. Ullrich, Phys. Rep. 337, 493 (2000).

${ }^{8}$ U. Schwengelbeck, L. Plaja, L. Roso, and E. Conejero Jarque, J. Phys. B 33, 1653 (2000).

${ }^{9}$ D. F. Zaretsky, Ph. A. Korneev, S. V. Popruzhenko, and W. Becker, J. Phys. B 37, 4817 (2004).

${ }^{10}$ R. A. Molina, D. Weinmann, and R. A. Jalabert, Phys. Rev. B
65, 155427 (2002).

${ }^{11}$ U. Kreibig and M. Vollmer, Optical Properties of Metal Clusters (Springer, New York, 1995).

${ }^{12}$ B. Lamprecht, J. R. Krenn, A. Leitner, and F. R. Aussenegg, Phys. Rev. Lett. 83, 4421 (1999).

${ }^{13}$ T. Sako, P.-A. Hervieux, and G. H. F. Diercksen, Phys. Rev. B 74, 045329 (2006).

${ }^{14}$ R. Balescu, Equilibrium and Nonequilibrium Statistical Mechanics (Wiley, New York, 1975).

${ }^{15}$ G. Manfredi and F. Haas, Phys. Rev. B 64, 075316 (2001).

${ }^{16}$ A. Banerjee and M. K. Harbolaa, J. Chem. Phys. 113, 5614 (2000).

${ }^{17}$ A. Domps, P.-G. Reinhard, and E. Suraud, Phys. Rev. Lett. 81, 5524 (1998).

${ }^{18}$ M. Brewczyk, K. Rzazewski, and C. W. Clark, Phys. Rev. Lett. 78, 191 (1997).

${ }^{19}$ G. Manfredi and P.-A. Hervieux, Phys. Rev. B 70, 201402(R) (2004).

${ }^{20}$ G. Manfredi and P.-A. Hervieux, Phys. Rev. B 72, 155421 
(2005).

${ }^{21}$ G. Manfredi and P.-A. Hervieux, Opt. Lett. 30, 3090 (2005).

${ }^{22}$ X. Liu, R. Stock, and W. Rudolph, Phys. Rev. B 72, 195431 (2005).

${ }^{23}$ G. Manfredi, Fields Inst. Commun. 46, 263 (2005).

${ }^{24}$ F. Haas, G. Manfredi, and M. R. Feix, Phys. Rev. E 62, 2763 (2000); F. Haas, L. G. Garcia, J. Goedert, and G. Manfredi, Phys. Plasmas 10, 3858 (2003); F. Haas, ibid. 12, 062117 (2005); M. Marklund, B. Eliasson, and P. K. Shukla, Phys. Rev. E 76, 067401 (2007); G. Brodin, M. Marklund, B. Eliasson, and P. K. Shukla, Phys. Rev. Lett. 98, 125001 (2007).

${ }^{25}$ S. H. Glenzer, O. L. Landen, P. Neumayer, R. W. Lee, K. Widmann, S. W. Pollaine, R. J. Wallace, G. Gregori, A. Höll, T. Bornath, R. Thiele, V. Schwarz, W.-D. Kraeft, and R. Redmer, Phys. Rev. Lett. 98, 065002 (2007).

${ }^{26}$ E. P. Wigner, Phys. Rev. 40, 749 (1932).
${ }^{27}$ V. I. Tatarskii, Usp. Fiz. Nauk 139, 587 (1983) [Sov. Phys. Usp. 26, 311 (1983)].

${ }^{28}$ M. Hillery, R. F. O'Connell, M. O. Scully, and E. P. Wigner, Phys. Rep. 106, 121 (1984).

${ }^{29}$ P. Hohenberg and W. Kohn, Phys. Rev. 136, B864 (1964); W. Kohn and L. J. Sham, Phys. Rev. 140, A1133 (1965).

${ }^{30}$ L. Brey, J. Dempsey, N. F. Johnson, and B. I. Halperin, Phys. Rev. B 42, 1240 (1990).

${ }^{31}$ A. G. Eguiluz, D. A. Campbell, A. A. Maradudin, and R. F. Wallis, Phys. Rev. B 30, 5449 (1984).

${ }^{32}$ M. Anderegg, B. Feuerbacher, and B. Fitton, Phys. Rev. Lett. 27, 1565 (1971).

${ }^{33}$ W. Bao, S. Jin, and P. A. Markowich, SIAM J. Sci. Comput. (USA) 25, 27 (2003).

${ }^{34}$ G. Vignale and W. Kohn, Phys. Rev. Lett. 77, 2037 (1996). 